\title{
El derecho a la salud como dimensión de la ciudadanía social en América Latina y Venezuela
}

The Right to the Health like Dimension of the Social Citizenship in Latin America and Venezuela

Andy del Valle Delgado Blanco

Universidad Central de Venezuela, Venezuela

andydelgadob@gmail.com

ORCID: http://orcid.org/0000-0002-1528-1285
DOI: https://doi.org/Javeriana.vj137.dsdc Redalyc: http://www.redalyc.org/articulo.oa $? \mathrm{id}=82556549005$

Fecha de recepción: 29 Mayo 2017

Fecha de aprobación: 04 Abril 2018

Fecha de publicación: 30 Noviembre 2018

\section{Resumen:}

Este artículo es parte de una investigación más rigurosa realizada por la autora para obtener el grado de doctora en Estudios del Desarrollo. Su propósito es examinar la realización efectiva del derecho a la salud como dimensión básica de la ciudadanía social, tanto en América Latina como en Venezuela. Para cumplir ese objetivo, la discusión se ha estructurado en tres partes: revisión del proceso de construcción de la ciudadanía social en la región, luego, en Venezuela y, finalmente, el estado en que se encuentra el disfrute efectivo de ese derecho en este último país. La revisión documental realizada sugiere que el desarrollo histórico de la ciudadanía social en esta parte del mundo evidencia ciclos de inclusión-exclusión en los que hay, por un lado, una expansión de derechos y mejoramiento de la calidad de vida de las personas, en términos globales y, por el otro, una desigualdad social que disminuye sus capacidades para desarrollarse, con pocas posibilidades de que las competencias y facultades intersubjetivas de los derechos traduzcan la igualdad normativa en un ejercicio más pleno del derecho. Así, el derecho a la salud, aunque tiene una concepción universalista, no se concreta en los hechos. Palabras claves Ciudadanía social; derechos; derecho a la salud

Palabras clave: Ciudadanía social, derechos, derecho a la salud.

\begin{abstract}
:
This article is a part of a more rigorous research realized by the authoress to obtain the Doctor's degree in Studies of the Development. His purpose is to examine the degree of effective realisation of the right to the health as basic dimension of the social citizenship, both in Latin America and in Venezuela. In order to achieve that objective the discussion has been structured in three parts: Firstly, Review of the process of construction of the social citizenship in the region, then in Venezuela and finally, the condition in which one finds the guarantee and effective enjoyment of this right in the latter country. The documentary review suggests that the historical development of the social citizenship in this part of the world demonstrates cycles of incorporation exclusion in that it is, on the one hand, an expansion of rights and improvement of the quality of life of the persons, in global terms and, for other one, a social inequality that limits their own abilities to develop, with few possibilities of that the competencies and intersubjective powers of the rights translate the normative equality in a fuller realization of the right. This way, the right to the health though it has a conception of universality it does not materialize.
\end{abstract}

Keywords: Social citizenship, rights, rights to the health.

\section{A manera de introducción}

En 1949, en el marco de un ciclo de conferencias en honor al economista Alfred Marshall, organizadas en la Universidad de Cambridge, el sociólogo THOMAS HUMPHREY MARSHALL presentó el análisis histórico-sociológico Citizenship and Social Class ${ }^{1}$, que marcó un hito en el estudio de la ciudadanía. Sin apartarse de la concepción clásica, sostuvo que esta es un estatus que se otorga a quienes son miembros de pleno derecho de una comunidad y que trae aparejados derechos y obligaciones ante la ley.

THOMAS HUMPHREY MARSHALL trazó una línea progresiva, en tiempo y espacio, sobre el proceso de construcción de la ciudadanía a lo largo de la historia inglesa, desde la Revolución Industrial hasta el nacimiento del Estado Providencia, expresada en tres componentes y fases: civil, político y social y denominó 
como ciudadanía social, el derecho a participar de un mínimo de bienestar social por el hecho de ser parte de una comunidad.

La ciudadanía social está estrechamente vinculada con el bienestar social que se alcanza a través del disfrute de la seguridad social, el trabajo, la educación, la recreación, la vivienda y la salud; derechos que requieren espacios, instituciones y oportunidades para su realización efectiva. En la medida en que un sector de la población deja de acceder a esa cuota de bienestar, su capacidad para ejercer los derechos de los que es titular disminuye: quienes son excluidos no pueden ser considerados verdaderos ciudadanos.

Pocas dimensiones evidencian el avance alcanzado por un país en la construcción de ciudadanía como la salud; precisamente, en este derecho se hacen patentes las desigualdades e inequidades y se muestra el nivel de vida alcanzado por las personas; el acceso oportuno y la disponibilidad de los servicios públicos de salud, entre otros elementos, configuran el bienestar ciudadano. Un niño sano es el germen de un ciudadano habilitado y con oportunidades reales de elegir y ser elegido.

El estado de la cuestión sobre la ciudadanía social y la concreción de sus habilitaciones o dimensiones, los llamados derechos sociales, tiene pertinencia y vigencia en el mundo del derecho y las ciencias sociales al centrarse en la realización efectiva de los derechos ciudadanos más allá de los aspectos formales; de allí que examinar las oportunidades reales que tienen los ciudadanos latinoamericanos y, en particular los venezolanos, para ejercer su ciudadanía social, teniendo como marco de referencia el derecho a la salud, es el objetivo que guía la discusión que se plantea en las páginas siguientes.

\section{Construcción de la ciudadanía social en América Latina}

En América Latina, el proceso de construcción de la ciudadanía social ha respondido a las particularidades y diferencias de los distintos países que la integran, y se ha apartado del curso lineal descrito por MARSHALL para Inglaterra. La región muestra una notable preeminencia del debate y el reconocimiento formal de los derechos sociales sobre los civiles y políticos. Los primeros irrumpen progresivamente en el escenario de la posguerra con la apertura del proceso de industrialización que exigía mano de obra sana y productiva, para contribuir a los planes de expansión de los distintos países. Los segundos presentan un desarrollo tardío, explicable, en gran medida, por tratarse de un subcontinente plagado para entonces por un amplio número de gobiernos autoritarios, con escasas experiencias en ejercicios democráticos y alternancias del poder ${ }^{2}$.

En términos reales, la instrumentación de los derechos sociales (como seguridad social y salud) en la región fue desigual y alcanzó, únicamente, a los trabajadores dependientes, y excluyó a los campesinos y a los que no estaban insertos en el mercado de trabajo formal; con lo cual — pese a ir contando con estructuras constitucionales y legales que apuntalaban los derechos sociales - las grandes mayorías quedaron excluidas del sistema. Los beneficios sociales derivados del sostenido crecimiento económico que desde mediados del siglo XX y hasta bien entrados los años setenta experimentó la región tuvieron cierto carácter redistributivo, sobre todo para esos trabajadores, al punto de que las políticas sociales instrumentadas, sobre todo en la década de los ochenta, actuaron más como forma de reestratificación para este sector que como universalización de derechos sociales.

Más tarde, esos mecanismos mostraron fisuras; así, en la llamada década perdida, el proceso de avance hacia la universalización de los derechos sociales — con matices según los países- sufrió notables contratiempos. Por un lado, las políticas de bienestar empezaron a limitarse en cuanto a acceso, cobertura y calidad de los servicios; por el otro, se desregularizaron y flexibilizaron los mercados de trabajo, se reformaron los sistemas de seguridad social, aumentaron la deuda y la crisis fiscal.

Frente a ese panorama se aplicaron medidas de ajuste que trajeron como consecuencia elevados costos sociales que los gobiernos de la región intentaron paliar, a través de programas compensatorios y focalizados, de corto plazo; dirigidos especialmente a los sectores más desposeídos, que se convirtieron en los más 
afectados por esas medidas. La acción de los poderes públicos, en materia social, pasó a ser vista como un asunto de "residualización", en el que las políticas y programas sociales se dirigían exclusivamente a quienes eran "incapaces de contratar su propia previsión social en términos privados" ${ }^{3}$.

Ya en el siglo XXI, sobre todo en esta última década, en el marco de la heterogeneidad que caracteriza a América Latina, la situación social y económica de sus países ha ido variando, con avances y retrocesos observables. Para 2011, de manera inédita en su historia, la clase media fue más numerosa que los sectores pobres y, entre 2013 y 2014, las tasas promedio regionales de pobreza e indigencia se mantuvieron estables. La primera se situó en el 28,2\% y la segunda, en el 11,8\% del total de la población: el número de personas pobres alcanzó los 168 millones; de ellos, 70 millones estaban en situación de indigencia ${ }^{4}$. Para 2015, el crecimiento económico anual del $40 \%$ más pobre de la población alcanzó un promedio del 5,2\%, con el que "eclipsó el desempeño de ese mismo grupo en las demás regiones del mundo". No obstante, "unos 75 millones de personas aún viven con menos de US\$2,50 al día, y casi las dos terceras partes de la población de la región son pobres o pertenecen a sectores vulnerables, que pueden caer en la pobreza" 5 .

Un examen particularizado de estos resultados globales evidencia la diversidad que acompaña a los países de la región ${ }^{6}$; mientras que los datos sobre Uruguay $(-14,9 \%)$, Perú $(-9,8 \%)$, Chile $(-9,1 \%)$, Colombia $(-6,4)$, Bolivia $(-6,3)$ y Brasil $(-7,9 \%)$ muestran una significativa caída de la tasa de pobreza; mientras México y Venezuela exhiben una tendencia al ascenso, a un ritmo anual que oscila entre $2 \%$ y casi $5 \%$. Por otro lado, aunque se esperaba que para 2015, 175 millones de personas estuvieran en situación de pobreza por ingresos ( 75 millones de ellas en indigencia) y que el crecimiento de la región caería a $0,4 \%$, los pronósticos indicaban que Bolivia y Perú crecerían cerca de un $4 \%$ o más ${ }^{7}$. ALICIA BÁRCENA, secretaria ejecutiva de la Comisión Económica para América Latina y el Caribe, CEPAL, en el marco de la reunión anual del Foro Económico Mundial 2016, señaló que, aun cuando la brecha en materia de distribución de los ingresos recibidos ha disminuido, América Latina es "la región más desigual del mundo" seguida de los países subsaharianos ${ }^{8}$; así, durante 2014, el 10\% más rico de su población amasó el $71 \%$ de la riqueza del subcontinente ${ }^{9}$.

La complejidad que trae aparejada la diversidad regional se expresa igualmente en el caso de la salud. Los indicadores de mortalidad infantil, prevalencia de ciertas enfermedades y expectativas de vida, en su conjunto, visibilizan en este segundo milenio "nuevos y viejos desafíos"; los primeros están asociados a factores que van desde las dinámicas demográficas y epidemiológicas, hasta considerar las tecnológicas, lo que requiere tanto prestaciones y tratamientos inéditos, como incrementos en costos y gastos. Otros retos no son novedosos y están relacionados con las "carencias históricas que presenta la región en materia de equidad de acceso real a servicios de salud oportunos y de calidad, la escasez de recursos humanos y financieros y los problemas de articulación de los sistemas" ${ }^{10}$. Esto plantea algunas interrogantes sobre la prestación universal de servicios de salud y requiere acuerdos específicos relativos a la asignación de recursos y a las políticas a instrumentarse.

Según reportes de la Organización Panamericana de la Salud, para finales del siglo XX, el promedio de vida, globalmente considerado, había aumentado en 18 años, desde hacía cuatro decenios y la región mostraba un "perfil singular de mortalidad que refleja una polarización epidemiológica" definido "por la coexistencia prolongada de dos patrones de mortalidad"; en general, la mortalidad en los primeros años de vida había descendido notablemente, aun cuando no podía decirse que la intensidad y la rapidez de esa disminución eran iguales en todos los países e incluso, se señalaba que esa desigualdad podía encontrarse en diferentes zonas de un mismo país ${ }^{11}$.

Entre 1999 y 2015, países como México, Perú y Brasil lograron disminuir en la mitad o más los porcentajes de la mortalidad infantil; en cambio, Panamá, Ecuador, Colombia, Venezuela, Argentina, Chile y Uruguay, aunque ofrecen mejoras en la materia, no se acercan a esos porcentajes ${ }^{12}$. En términos globales, desde lo cuantitativo y lo cualitativo, la región ha logrado significativos avances en la reducción de la mortalidad infantil; las tasas en esta materia "han descendido en la mayoría de los países a menos de 30 defunciones por 1.000 nacidos vivos, y, en algunos otros son similares a las del mundo desarrollado"; incluso, el perfil de las 
causas de la mortalidad infantil se ha modificado, cada vez son menos las infecciones intestinales y respiratorias 13

Frente a los progresos señalados, las desigualdades en las oportunidades se mantienen y el ejercicio de los derechos enfrenta serias limitaciones. Algunos reportes indican que, pese a las evidentes mejorías, "el promedio de mortalidad infantil se sitúa en un valor de 18 muertes por mil nacidos vivos" lo que es "casi 5 veces superior al promedio de los países desarrollados", y, por otra parte, en países como Bolivia y Colombia, "los niños pobres tienen de 2 a 5 veces mayor probabilidad de morir en el primer año de vida que los niños ricos" 14 .

El impacto que tienen los "gastos de bolsillo" en materia de salud también revelan esas desigualdades 15 , debido a sus consecuencias en los hogares pobres, sobre todo cuando hay adultos mayores y niños entre sus miembros. La evolución de ese gasto "en siete países de América del Sur revela un incremento de forma agregada y per cápita entre 2000 y 2011". Este incremento fue constante en "Brasil, Colombia, Ecuador y Chile; Venezuela tiene el gasto más alto y su participación en el gasto total — público y privado— en salud es también el más elevado". Mientras que Uruguay, Bolivia, Perú, México y Panamá mostraron una tendencia a la disminución ${ }^{16}$.

Otros datos, distintos a los anteriores, también aportan información significativa sobre la protección efectiva del derecho a la salud, en la región, para los años arriba referidos. Así, las tasas de prevalencia del VIHSida, en la población de 15 a 24 años han disminuido en países como Perú, Colombia, Ecuador, México, y Panamá y aumentado en Uruguay, Chile, Bolivia y Venezuela ${ }^{17}$.

Tradicionalmente, las desigualdades se han asociado a la distribución de los ingresos y las rentas; no obstante, también se acude a ese término para describir el contexto social. Hay desigualdad cuando las personas reciben tratamientos o beneficios distintos, bien sea desde lo legal o material, en función de categorizaciones que se hagan o, cuando se las discrimina y no pueden acceder a ese bienestar material del que hablaba MARSHALL; como lo refiere la OEA, esta situación es de naturaleza durable, no transitoria ni incidental ${ }^{18}$.

Las inequidades y desigualdades (cualesquiera de las que se trate) son rémoras para el desarrollo y el ejercicio de los derechos, sobre todo de los sociales y, muy especialmente, el de la salud. Los pobres suelen ser también los que menos acceso tienen a la atención en salud y, como lo señala AMARTYA SEN ${ }^{19}$, esta dimensión es fundamental para entender que las personas puedan hacer lo que quieren y lo que valoran, dentro de lo cual, los bienes y servicios (en salud) pueden ser determinantes. La ampliación de las capacidades de las personas pasa por tener un estado de salud que permita no solo las realizaciones básicas de la vida, sino los contenidos vinculados con el desarrollo de un mayor potencial del ser humano y por lo tanto, con las garantías de una ciudadanía social más efectiva y, por ende, de un ejercicio efectivo del derecho.

Comúnmente, se acepta que la característica definitoria de los derechos sociales, en los Estados latinoamericanos, incluido el colombiano y el venezolano, ha sido su existencia en el marco formal, reconocida en algunos casos, primero, en los textos legales, para luego, consagrarse en las cartas constitucionales; sin embargo, en el terreno real, en el día a día, en la posibilidad existencial de su ejercicio ciudadano, lo que ha existido es una gran segmentación que ha limitado su plena efectividad.

A criterio de LUIS GÓMEZ-CALCAÑO, la institucionalización de la ciudadanía en América Latina, durante gran parte del siglo XX, gravitó en torno a tres características principales. La primera de ellas estaba relacionada con la naturaleza de los derechos que "aparecen como una dádiva de un líder o de un partido de vanguardia” y, por tanto, los sujetos de derecho no lograban constituirse autónomamente respecto al líder carismático o el Estado. La segunda característica se vinculaba con la jerarquización de los derechos que constituyen la ciudadanía, lo que se evidencia en que los derechos civiles se mantuvieron rezagados frente a los derechos políticos y los sociales y, la tercera y última, tenía qué ver con los efectos que esa institucionalización tuvo en la vida de los ciudadanos. Esta línea argumentativa de GÓMEZ-CALCAÑO pretende mostrar, por 
una parte, que hubo grupos sociales que no fueron incorporados al consumo y por la otra, que la brecha de las desigualdades sociales no había disminuido ${ }^{20}$.

Pasados más de veinte años, el argumento de GÓMEZ-CALCAÑO mantiene su vigencia. Las particularidades existentes en muchos países latinoamericanos le otorgan a la ciudadanía social de la región un patrón clientelar; exacerbado por su dependencia de un Estado proteccionista que ha configurado unos derechos sociales, cuyo significado es entendido como "algo que se otorga desde fuera y se ha olvidado el trabajo de conquistarla desde dentro, desde el sujeto que se conquista a sí mismo recuperando su subjetividad, su condición de sí como hombres y mujeres que habitan el mundo en el que realizan su vida, su existencia" ${ }^{21}$ . En este sentido, vale la pena detenerse en el caso venezolano.

\section{Ciudadanía social en Venezuela. Proceso de construcción}

En Venezuela, el ejercicio de trazar la línea histórica del proceso de ampliación de la ciudadanía, aludido por MARSHALL, tiene como punto de partida la mitad del siglo XIX y se prolonga a lo largo del siglo XX, con difusos interludios de retracción. El primer hito real de ese proceso se dio el 24 de marzo de 1854, cuando el presidente José Gregorio Monagas promulgó la Ley de Abolición de la Esclavitud ${ }^{22}$ y la igualdad pasó a ser un concepto universal en el país, al darle amplitud material y formal a la libertad, dimensión intrínseca de la ciudadanía civil. El segundo marcador se ubica en el siglo XX con el reconocimiento y ampliación de los derechos políticos: la Constitución de 1945 extiende el derecho al sufragio, bajo ciertas condiciones, a las mujeres ${ }^{23}$; y la Constitución de 1947 consagra el sufragio universal como un derecho constitucional ${ }^{24}$.

La cuestión social empezó a ser considerada en las agendas públicas, ya bien entrado el siglo XX. En 1936, el general ELEAZAR LÓPEZ-CONTRERAS impulsó el Programa de Febrero en el que se focaliza la acción estatal para resolver las carencias en materia laboral, asistencia social, higiene pública y educación; se creó un cuerpo normativo en torno a la seguridad social y, colateralmente, una red institucional para dar soporte a esos derechos. El 16 de julio de ese mismo año le es puesto el Ejecútese a la Constitución y a la Ley del Trabajo; esta última, de singular avance para la época, puso al país a la vanguardia legislativa en esa materia en el mundo; mantuvo su vigencia alrededor de sesenta años. En 1940 se asentó una nueva concepción jurídico-normativa expresada en el concepto de seguridad social que perseguía darle seguridad al trabajador frente a los riesgos, para lo cual se promulgó la Ley del Seguro Social.

En 1947, producto de la segunda Asamblea Constituyente del siglo XX ${ }^{25}$, vio la luz la primera Constitución democrático-social de Venezuela e instituyó de manera expresa y formal los derechos sociales, con un enfoque fundamentado en un modelo societario igualitario, sobre la base de la justicia social ${ }^{26}$. Este texto constitucional, cuya vigencia fue de apenas un año, debido a la toma del poder por los militares 27 , prevé, por vez primera en la historia constitucional republicana de Venezuela, derechos laborales como la contratación colectiva, la huelga, la estabilidad laboral y las prestaciones sociales; también se instituyeron los derechos a la salud y a la seguridad social, se retomó el de la educación desaparecido como tal desde la Constitución con la que nació la República en $1811^{28}$.

Años más tarde, en 1961, ya restituido el orden constitucional se promulgó una Constitución que rescató el modelo igualitario de sociedad de 1947 y configuró de manera implícita el Estado Social de Derecho, al preservar los derechos sociales allí establecidos, sobre todo en materia de educación, salud y seguridad social. Durante al menos las dos décadas siguientes el sistema satisfizo — parcialmente - las demandas sociales de los ciudadanos. Hubo mejoras significativas en índices sociales como alfabetización, escolarización, consumo calórico y talla; en materia de salud fue creada una vasta red asistencial a la cual tuvo acceso - de manera desigual, en cuanto a cobertura y calidad - gran parte de la población. La representativa mejoría de las condiciones de vida de la población generó grandes expectativas de movilidad social y económica, derivadas 
del hecho de vivir en un sistema democrático que aupaba un desarrollo económico basado en la renta petrolera, donde había posibilidades de bienestar para gran parte de sus ciudadanos.

Ese proceso de construcción de la ciudadanía social iniciado casi a mediados del siglo XX, marcado por avances y retrocesos, respondió a unas características y dimensiones específicas propias de lo que MARIO DOS SANTOS ${ }^{29}$ denomina el Protoestado de Bienestar Latinoamericano o del Estado Social delineado en la Constitución venezolana de 1961, sobre la base de la protección social de los trabajadores asalariados y la asistencia social. A finales de los años setenta, Venezuela empezó a mostrar los primeros desajustes socioeconómicos y los incipientes rasgos de deformación del aparato estatal, que se agudizaron en los ochenta y evidenciaron la desarticulación existente entre los mecanismos de conciliación y la estructura burocrática institucional ${ }^{30}$. En materia de salud, el modelo institucional lucía desconectado, disperso, agotado e incapaz de impactar en las condiciones de vida de la mayoría de la población al limitarse a las actividades curativas e individuales, con un escaso radio de acción hacia lo preventivo-colectivo ${ }^{31}$.

En la medida en que la situación se fue complejizando, debido a factores como el mal manejo de los recursos del Estado, el clientelismo político, el crecimiento demográfico y la crisis económica, surgieron debates sobre la viabilidad de las normas constitucionales que consagraban los derechos sociales. La creciente idea de que el Estado no estaba en condiciones de seguir haciendo frente a las demandas sociales de la población coadyuvó al escepticismo de los sectores populares, que les retiraron su apoyo a los partidos políticos tradicionales, hasta entonces mediadores entre el Estado y la sociedad. Este escenario sirvió de marco al estallido social de febrero de 1989, constituido por violentas manifestaciones y saqueos, conocidas como el "Caracazo", que de acuerdo a los expertos tuvieron como causas inmediatas los aumentos del transporte urbano y el deterioro de las condiciones de vida de los sectores populares ${ }^{32}$.

La situación política, social y económica del país continuó agravándose; en lo político y social, el país asistió a dos intentonas golpistas luego de más de 30 años de vida democrática, seguido de la renuncia del presidente de la República CARLOS ANDRÉS PÉREZ luego de que el máximo tribunal de la República lo suspendió de sus funciones para ser enjuiciado por peculado y malversación de fondos públicos, vino un interludio con un mandatario provisional para llegar a un proceso electoral pleno de cuestionamientos y ofrecimientos de mejorar la vida de la ciudadanía ${ }^{33}$. No obstante, un par de años después de ganar las elecciones, el nuevo presidente inició una política económica de ajustes, bajo los lineamientos de los organismos internacionales que requirieron la instrumentación de políticas de corte social, focalizadas y compensatorias para resguardar la estabilidad social mientras durara el ajuste ${ }^{34}$.

En materia de salud se apreciaron notables contradicciones; por un lado, hubo un muy moderado avance del proceso de descentralización y transferencia de competencias a los gobiernos regionales y locales que no siempre fue abanderado por los responsables del Despacho de Salud. Por otra parte, se instrumentaron medidas que llevaron al cobro directo o indirecto en los establecimientos públicos de salud, se incrementaron los seguros privados de hospitalización, cirugía y maternidad de los trabajadores del sector público, se otorgaron estímulos y facilidades económicas para la construcción de centros de salud privados y hubo una reducción del cupo de las universidades para la formación de profesionales de la salud ${ }^{35}$.

En medio de ese panorama "...la ciudadanía social no era justamente un elemento importante de las políticas que se limitaban a indemnizar los daños de las políticas de ajuste..." ${ }^{36}$. La tendencia era a considerar los derechos sociales como una responsabilidad individual de los ciudadanos quienes debían autosatisfacerlos, conforme a las fuerzas del mercado. La crisis que se abatió sobre el país, asociada al desaliento y a la pérdida de la ilusión colectiva de lo que GÓMEZ-CALCAÑO ${ }^{37}$ llamó una visión de futuro, influyó en la búsqueda de propuestas de cambio lo que permitió, en diciembre de 1998, la llegada al poder, por vía electoral de HUGO CHÁVEZ-FRÍAS, quien seis años antes, había sido la cabeza visible del primer intento de derrocamiento del entonces presidente constitucional de la república, Carlos Andrés Pérez. 
La promesa electoral que marcó la campaña del candidato Chávez Frías fue la convocatoria de una Asamblea Constituyente que sentara las bases de una nueva República, lo que efectivamente hizo el mismo día de su juramentación como presidente al firmar el Decreto Presidencial $\mathrm{N}^{\circ} 3$, para "la realización de un referendo para que el pueblo se pronunciara sobre la convocatoria de una Asamblea Nacional Constituyente" 38 .

Como resultado del proceso constituyente se promulgó la Constitución de la República Bolivariana de Venezuela ${ }^{39}$ en la que por una parte, se amplió el rango de los derechos sociales que constituyen la ciudadanía social y se reivindicó su concepción redistributiva creándose "un nuevo imaginario en el que estos derechos son exigibles al tener el estatus de derechos humanos" ${ }^{40}$ y, por la otra, se propusieron cambios institucionales y normativos de gran amplitud. Con esta Constitución, el Estado venezolano asumió la responsabilidad de garantizar las obligaciones sociales y la procura de la justicia social al consagrar de manera expresa e inédita, en la historia constitucional de Venezuela, el Estado Social de Derecho.

\section{Garantía y ejercicio del derecho a la salud en Venezuela. Oportunidades reales}

Los elementos que influencian los significados que los ciudadanos otorgan a sus derechos van más allá de la protección postulada en las normas, de los sujetos responsables de garantizarla, de a quiénes se dirija, de sus fines, medios y alcances; son las oportunidades reales para su ejercicio y disfrute, las opciones con las que se cuentan, la accesibilidad, calidad y bienestar que proporcionan los servicios y bienes a través de los cuales se realiza el derecho, los que le aportan sentido y contenido.

Para AMARTYA SEN ${ }^{41}$ solo puede hablarse de seres humanos libres cuando el medio en el que se desenvuelven les proporciona oportunidades suficientes para desarrollar o potenciar sus capacidades, lo que está muy relacionado con el control que tiene la persona sobre los mecanismos económicos, políticos y sociales que ofrece el sistema legal. Son estas oportunidades — capacidades - las que hacen posible el desarrollo y por ende, la construcción de ciudadanía.

La Constitución de 1999, además de centrar en el Estado la garantía de la salud, establece en sus artículos 83, 84 y 85 los elementos básicos para cumplir con esa atribución: a) la promoción y desarrollo de políticas orientadas a elevar la calidad de vida, el bienestar colectivo y el acceso a los servicios; b) la creación, ejercicio y rectoría de un sistema público nacional de salud que enfatice la promoción de la salud, la prevención de enfermedades y el tratamiento oportuno y la rehabilitación; y, c) el desarrollo de políticas destinadas a la formación de profesionales en la materia y a desarrollar una industria nacional de producción de insumos para la salud.

Cuando se revisa el grado de cumplimiento de los deberes constitucionales por parte del Estado venezolano se descubren señales que evidencian menoscabo del ejercicio del derecho y disminuyen las oportunidades reales de los ciudadanos. Indicadores que pueden ser medidos al revisar el estado de la infraestructura sanitaria y los equipos médico-quirúrgicos, las condiciones de trabajo del personal de salud, el funcionamiento de los servicios de salud, el tipo, calidad y oportunidad de los tratamientos y la dispensa de los medicamentos, entre otros factores. Los expertos en el tema ${ }^{42}$, los medios de comunicación e incluso las más altas autoridades del país han admitido, de una forma u otra, el deterioro de la situación de la salud en Venezuela. En 2009, lo hizo el entonces presidente Chávez ${ }^{43}$; más tarde, el 3 de marzo de 2013, el vicepresidente Nicolás Maduro afirmó públicamente ${ }^{44}$ que muchos hospitales del país se encontraban en una "situación bastante deplorable" y, en diciembre de ese mismo año, ya investido como Presidente de la República recalcó la necesidad de "recuperar los viejos hospitales públicos, cuyo estado calificó como \#una vergüenza para la Revolución\#” 45 .

En lo que se ha llamado el sistema de salud venezolano ${ }^{46}$ existe una alta fragmentación de entes, recursos y sistemas de atención, en general, paralelos, independientes uno del otro y bajo la autoridad de órganos 
diferentes; el Ministerio del Poder Popular para la Salud regula la red de atención primaria, mientras que el Instituto Venezolano de los Seguros Sociales se ocupa de los trabajadores asegurados; y la Misión Barrio Adentro, de una parte de la atención primaria, por solo citar tres ejemplos relevantes. Otro aspecto que cabe resaltar es la insuficiencia de recursos humanos en el área, bien porque las escuelas de medicina gradúan a pocos profesionales o especialistas o porque, los profesionales con alguna especialidad una vez terminada la residencia migran del sistema público al privado o hacia mejores plazas en el extranjero, lo que reduce la capacidad de dar respuesta al crecimiento de la demanda en materia de asistencia sanitaria ${ }^{47}$.

Los cuerpos colegiados que agrupan a los sanitaristas, epidemiólogos y otras especialistas en salud pública han venido reportando el resurgimiento de enfermedades que se creían desaparecidas y volvieron a emerger en los últimos años ${ }^{48}$. En este sentido, la Sociedad Venezolana de Salud Pública, SVSP, reportó que para 2010, en un brote que duró "más de 16 meses", el dengue alcanzó la cifra "histórica máxima de incidencia acumulada 124.931 casos con una tasa de incidencia anual acumulada de $433 \times 100.000 \mathrm{~h}$.; de casos graves 10.278 y de muertes 124 (estimado) que afectó todo el país". Según ese mismo trabajo la malaria alcanzó en 2013: "cifras récord de incidencia de casos 76.621 y tasa de 281,4 x $100.000 \mathrm{~h}$., desde que se llevan registros epidemiológicos de la enfermedad con 50,1\% de aumento al comparar con el año 2012" 49 .

El Boletín Epidemiológico emanado del Ministerio del Poder Popular para la Salud, fuente oficial del Estado venezolano en la materia ${ }^{50}$, aporta evidencias sobre los particulares reseñados. En la semana 13, del primer trimestre de 2010, se notificaron 1.088 casos probables de dengue, siendo que la Tasa Nacional de Incidencia acumulada para esa semana fue de 76.8 por 100.000 habitantes, la cual era superada por 9 estados. En el caso de la malaria, enfermedad erradicada en gran parte del mundo actual, se reportó un acumulativo de 12.717 casos; para la semana 14 de 2014 el acumulado fue 75.020 casos (dengue) y el índice epidémico fue de 164, lo que representa un incremento de 64\% "con relación a la mediatriz 2009-2013". Hasta esa fecha existía "un acumulativo de 73.572 casos con origen de infección en el país", lo que representaba un "aumento de $12 \%$ con respecto al período homólogo del año anterior".

Los expertos coinciden en señalar que en los últimos años se ha debilitado el enfoque preventivo y se ha enfatizado el médico-curativo. Un ejemplo vinculado a esta situación es la baja cobertura en vacunas. Los programas de vacunación se han reducido y esto ha facilitado: "la reaparición de brotes epidémicos de enfermedades transmisibles, controlables mediante vacunas y el riesgo de aparición de otras con grave impacto epidémico como poliomielitis y difteria que fueron erradicadas hace muchos años" 51 .

Otra consecuencia del debilitamiento del enfoque preventivo se evidencia en las altas tasas de morbilidad asociadas al VIH-Sida que han ido en ascenso ${ }^{52}$; situación que pudiera estarse agravando, debido a las dificultades que enfrentan los pacientes para acceder, de manera efectiva y oportuna, a los retrovirales, conforme lo señalan diversas organizaciones de derechos humanos — entre ellas, PROVEA—, en sus informes de los últimos años.

Desde 2013, varias organizaciones de derechos humanos han venido reportando interrupciones en la entrega de tratamientos para enfermedades crónicas y la suspensión de la radioterapia para enfermos con cáncer, el déficit de especialistas para atenderlas y los medios para el diagnóstico temprano. Ya antes se habían encendido las alarmas para dar cuenta de las fallas en la calidad de atención, el desabastecimiento e insuficiencia de insumos y medicamentos, que se ha ido incrementando al punto de pasar "de desabastecimiento a escasez en productos esenciales". De acuerdo con diversos reportes, el deterioro de los equipos médicos y quirúrgicos se ha ido agravando progresivamente, así ya desde esas fechas "en los 10 principales centros hospitalarios de Caracas, 69\% de los quirófanos y 52\% de las camas no se encontraban

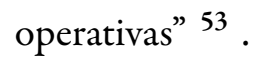

Las situaciones expuestas evidencian restricciones claras en el ejercicio efectivo del derecho a la salud. Hay un patrón ascendente que muestra tanto dificultades para la accesibilidad a establecimientos, bienes, servicios 
y programas de salud; como la no disponibilidad de insumos, tratamientos y medicamentos, y la desmejora de la calidad de los servicios.

\section{Conclusión}

Uno de los aspectos prioritarios a tomar en cuenta para mejorar la calidad de vida de los grupos humanos es la vinculación entre ciudadanía, derechos y oportunidades; en la medida en que la exclusión social se acrecienta, se socava el ejercicio de la ciudadanía, los excluidos son considerados como necesitados y beneficiarios de políticas sociales a través de un sistema clientelar, no como ciudadanos con deberes y derechos que deben ser realizados de manera efectiva.

El desarrollo histórico de la ciudadanía social en América Latina evidencia marcados ciclos de inclusiónexclusión, en los cuales la desigualdad social disminuye las capacidades de las personas para tener una vida mejor, sin posibilidades de que las competencias y facultades intersubjetivas de los derechos permitan un ejercicio democrático que traduzca la igualdad, en oportunidades reales.

En términos formales, la Constitución venezolana de 1999 amplía el rango de los derechos sociales con mecanismos de participación ciudadana para hacerlos efectivos, promueve la corresponsabilidad y la igualdad de todos los integrantes de la sociedad venezolana, con lo cual surgen nuevos actores y posibilidades. Se revaloriza la ciudadanía social como vía para la libertad de las personas, el Estado aparece como un propiciador de oportunidades en salud. El derecho a la salud mantiene una concepción universalista, en la cual la salud se tutela como parte del derecho a la vida y su promoción, defensa y gestión se convierten en un asunto de todos. Se insta a la construcción de políticas que respeten al ciudadano, como sujeto de derechos y permitan cambios en su calidad de vida, bienestar y procuren su acceso a los servicios.

En los hechos, en los últimos años, el desabastecimiento de medicamentos, el deterioro de la infraestructura sanitaria, las dificultades para la accesibilidad a equipos médicos y quirúrgicos, la baja calidad en la prestación de los servicios son factores que se conjuran para la contracción del ejercicio efectivo de una de las dimensiones de la ciudadanía social: el derecho a la salud.

\section{Bibliografía}

$\cdots$

\section{Libros}

CALDERA, RAFAEL, Mi carta de intención con el pueblo de Venezuela (Talleres Gráficos de Joaquín Ibarra/ Impresores, Caracas, 1993).

COMBELLAS, RICARDO, Una Constitución para el futuro. El debate constitucional en Venezuela (Fundación Konrad Adenauer, Caracas, 1994).

Comisión Presidencial para la Reforma del Estado, COPRE, Una politica social para la reafirmación de la democracia, volumen 8 (Editorial Arte, Caracas, 1989).

GONZÁLEZ, MARINO, Las politicas de salud en Venezuela: alternativas para su mejoramiento (Instituto Latinoamericano de Investigaciones Sociales, ILDIS, Caracas, 2008). Disponible en: http://ildis.org.ve/websit e/p_index.php?ids $=7 \&$ tipo $=$ P\&vermas $=131$

MARSHALL, THOMAS HUMPHREY \& BOTTOMORE, TOM, Ciudadania y clase social (Alianza Editorial, Madrid, 1998).

MÉNDEZ-CEGARRA, ABSALÓN, Estado, politica social y trabajo social en la Venezuela actual (Universidad Central de Venezuela, Facultad de Ciencias Económicas y Sociales, Unidad de Publicaciones, Caracas, 1992). 
SEN, AMARTYA, Desarrollo y libertad (Editorial Planeta, Barcelona, 2000).

\section{Capítulos de libro}

BARBA-SOLANO, CARLOS, Reforma social y ciudadania social en América Latina durante los años noventa: una perspectiva comparada, en Retos para la integración social de los pobres en América Latina, 51-84 (CARLOS BARBA-SOLANO, comp., Colección Grupos de Trabajo, Consejo Latinoamericano de Ciencias Sociales, CLACSO, Buenos Aires, 2009). Disponible en: http://bvsde.org.ni/clacso/publicaciones/Retosparalaintegrac ionsocial.pdf

BOLÍVAR, LIGIA \& PÉREZ-CAMPOS, MAGALY, El Sistema de Derechos Humanos en la Constitución de 1961 y Propuestas de Reforma, en El sistema politico venezolano: crisis y transformaciones, 33-129 (ÁNGEL EDUARDO ÁLVAREZ, coord., Universidad Central de Venezuela, UCV, Facultad de Ciencias Jurídicas y Políticas, Instituto de Estudios Políticos, Caracas, 1996).

CRUCES, GUILLERMO, Protección social y sistemas de salud, en Los sistemas de salud y de protección social frente a los nuevos escenarios epidemiológicos y demográficos, 77-111 (Reunión de Expertos sobre Población y Pobreza en América Latina y el Caribe, 14 y 15 de noviembre 2006, Santiago, Chile, División de Población de la Comisión Económica para América Latina y el Caribe, CELADE, con el auspicio del Fondo de Población de las Naciones Unidas, UNFPA, Santiago de Chile, 2006). Disponible en: https://www.cepal.org/sites/default/files/events/f iles/cruces2.pdf

GÓMEZ-CALCAÑO, LUIS, La redefinición del Estado Social: el caso de Venezuela, en El cambio del papel del Estado en América Latina, 285-316 (MENNO VELLINGA, coord., Siglo XXI Editores, México, 1997).

LACRUZ, TITO, Del antes al ahora: Balances de la política social en Venezuela, en Balance y perspectivas de la política social en Venezuela, 111-184 (THAIS MAINGON, coord., Instituto Latinoamericano de Investigaciones Sociales, ILDIS, Centro de Estudios del Desarrollo, Universidad Central de Venezuela Cendes, Fondo de Población de las Naciones Unidas, UNFPA, Caracas, 2006). Disponible en: http://ildis.org.ve/website/admin istrador/uploads/PoliticaSocial.pdf

O'DONNELL, GUILLERMO, Hacia un Estado de y para la democracia, en Democracia/Estado/Ciudadania hacia un Estado de y para la democracia en América Latina, 25-64 (RODOLFO MARIANI, coord., Programa de las Naciones Unidas para el Desarrollo, PNUD, Lima, 2008). Disponible en: http://www.iidh.ed.cr/multic/UserF iles/Biblioteca/IIDHSeguridad/12_2010/be846c2a-a0e6-44d0-9fae-5d9d637df9ff.pdf

DOS SANTOS, MARIO Estrategias de gobernabilidad en la crisis. Análisis comparado de las politicas de integración social en América Latina: fondos sociales de emergencia y tendencias a la focalización, en Desempleo estructural, pobreza y precariedad. Coordenadas y estrategias de politica social en la Argentina y América Latina, 243-267 (SUSANA PEÑALVA \& ALEJANDRO ROFMAN, comps., Centro de Estudios Urbanos y Regionales, CEUR, Ediciones Nueva Visión, Buenos Aires, 1996).

\section{Revistas}

AGUIRRE, ALEJANDRO \& VELA-PEÓN, FORTINO, Descenso y transición epidemiológica de la mortalidad infantil en América Latina y el Caribe, XLII Notas de Población, 110, 59-77 (2015). Disponible en: https://rep ositorio.cepal.org/bitstream/handle/11362/39373/1/03_Aguirre_101A.pdf

AIN-BILBAO, MARÍA LUCIANA, Ser ciudadano en América Latina: un status conflictivo, 1 Iberoamérica Global, The Hebrew University of Jerusalem, 4, 3-13 (2008).

ARIAS-MURILLO, FRANCISCO ANTONIO, Ciudadania en el contexto democrático de América Latina, 3 Hallazgos, Revista de Investigaciones, 5, 151-165 (2006). Disponible en: http://revistas.usta.edu.co/index.php/ hallazgos/article/view/1638/1791, http://www.usta.edu.co/

CUNAARR-CONDE, EDITH MABEL, Modernización reflexiva y democratización en Venezuela, 20 Opción, Revista de Ciencias Humanas y Sociales, 43, 119-142 (2004). Disponible en: http://produccioncientificaluz.org/index. php/opcion/article/view/6286/6274 
D'ELIA, JO, Situación de la salud en Venezuela. La escasez se ha extendido a 1.200 medicamentos, 769 Revista SIC, 388-391 (2014). Disponible en: http://gumilla.org/biblioteca/bases/biblo/texto/SIC2014769_388-391.pdf

GÓMEZ-CALCAÑO, LUIS, Ciudadania, politica socialy sociedad civil en América Latina, 36 Cuadernos del Cendes, Centro de Estudios del Desarrollo, Universidad Central de Venezuela, 11-34 (1997).

MOLINA, JOSÉ E., El proceso de consolidación de la hegemonia en Venezuela y sus consecuencias políticas, 9 Cuestiones Politicas, 73-81 (1992).

NARANJO, MARÍA, La politica de salud en Venezuela durante el periodo 1999-2012. Aportes para su formulación e implementación, 12 Comunidad y Salud, 2, 80-86 (2014). Disponible en: http://www.redalyc.org/pdf/3757/3 75740255011.pdf

NOSETTO, LUCIANO, Variaciones latinoamericanas en torno al concepto de ciudadanía, 6 Revista de Filosofia Factótum, 77-97 (2009). Disponible en: http://www.revistafactotum.com/revista/f_6/articulos/Factotum_6_ 6_Luciano_Nosetto.pdf

PÉREZ-LUGO, JORGE ERNESTO, La necesaria reforma de los Sistemas de Salud en América Latina, 13 Gaceta Laboral, 1, 43-57 (2007). Disponible en: http://produccioncientificaluz.org/index.php/gaceta/article/view/3 $586 / 3584$

SEN, AMARTYA, ¿Por qué la equidad en salud?, 11 Revista Panamericana de Salud Pública, 5/6, 302-309 (2002). Disponible en: http://iris.paho.org/xmlui/handle/123456789/8704?show=full

WALTER, CARLOS, A Strange Form of Declaring a Health Emergency: The Case of Venezuela, 55 World Medical Journal, 4, 157-159 (2009). https://www.wma.net/wp-content/uploads/2016/11/wmj24.pdf

\section{Informes, reportes}

Banco Mundial, Informe anual 2015 (Banco Mundial, Washington, D. C., 2015). Disponible en: http://documents.worldbank.org/curated/en/684241468187780194/pdf/99910-WBAR-Box393213B-P UBLIC-disclosed-10-2-15-SP.pdf

BÁRCENA, ALICIA, América Latina y el Caribe es la región más desigual del mundo ¿Cómo solucionarlo? Columna de opinión publicada en el marco de la reunión anual del Foro Económico Mundial (25 de enero de 2016). Disponible en: https://www.cepal.org/es/articulos/2016-america-latina-caribe-es-la-region-mas-desigual-mun do-como-solucionarlo, http://www.cepal.org/es/articulos

Cámara de Comercio de Maracaibo, Reflexiones sobre el Sistema de Salud Venezolano, Trabajo elaborado por un equipo interdisciplinario bajo el auspicio de la Cámara de Comercio de Maracaibo (2011). Disponible en: http://www.ccm.org.ve/archivos/documentos/REFLEXIONES\%20SOBRE\%20EL\%20SISTEMA\%20D E\%20SALUD\%20VENEZOLANO.pdf

Comisión Económica para América Latina y el Caribe, CEPAL, Panorama social de América Latina (CEPAL, Santiago de Chile, 2015). Disponible en: https://repositorio.cepal.org/bitstream/handle/11362/39965/S1600175_es.p $\mathrm{df}$

Federación Médica de Venezuela, FMV, Diagnóstico del sector salud en Venezuela: estudios de las enfermedades emergentes y reemergentes (Ponencia central presentada en la LXIII Reunión Ordinaria de la Asamblea, realizada en Punto Fijo, estado Falcón, 27 al 31 de octubre de 2008).

GONZÁLEZ-R., MARINO J., Gasto de bolsillo en salud en América Latina (1995-2013): evolución e implicaciones de politicas (Trabajo de incorporación como miembro correspondiente nacional puesto 39 de la Academia Nacional de Medicina, Caracas, julio de 2016). Disponible en: http://www.anm.org.ve/anm/cms/svcobtenerdocumento ssinlimitepdf.php?id=0000000127\& tipo=normal\&nn=Fhgfeaf

Ministerio del Poder Popular para la Salud, MPPS, Boletín epidemiológico (2010). Disponible en: https://www.ovsalu d.org/descargas/publicaciones/documentos-oficiales/Boletin-Epidemiologico-2010.pdf

Ministerio del Poder Popular para la Salud, MPPS, Boletín epidemiológico (2014). Disponible en: https://www.ovsalu d.org/descargas/publicaciones/documentos-oficiales/Boletin-Epidemiologico-2014.pdf 
Organización Panamericana de la Salud, OPS-PAHO, Análisis de situación de salud en las Américas, 1999-2000, 21 Boletín Epidemiológico, 4, 2-3 (2000). Disponible en: http://iris.paho.org/xmlui/bitstream/handle/123456789 /31866/be_v21n4.pdf?sequence=1\&isAllowed=y, http://cidbimena.desastres.hn/docum/ops/publicaciones

Oxfam, Privilegios que niegan derechos. Desigualdad extrema y secuestro de la democracia en América Latina y el Caribe (2015). Disponible en: https://www.oxfam.org/es/informes/privilegios-que-niegan-derechos

Programa Venezolano de Educación-Acción en Derechos Humanos, PROVEA, Informe Anual 2013: Situación de los derechos humanos en Venezuela (2013). Disponible en: https://www.derechos.org.ve/informe-anual/informe-a nual-enero-diciembre-2013

Sociedad Venezolana de Salud Pública, SVSP \& Red Defendamos la Epidemiología Nacional, Pequeñas picaduras, grandes amenazas. Breve relato de éxitos, fracasos y amenazas de la lucha contra las enfermedades vectoriales en Venezuela, Día Mundial de la Salud de 2014, 7 de abril de 2014, dedicado al control de las enfermedades transmitidas por vectores. Disponible en: https://www.bitacoramedica.com/wp-content/uploads/2014/05/SV SP-2014-6-65-X-87.pdf

\section{Tesis}

HERNÁNDEZ-CORTEZ, NOÉ, El discurso ideológico de la politica social en Venezuela. 1989-2010: un enfoque postestructuralista (Tesis doctoral, Facultad Latinoamericana de Ciencias sociales, FLACSO, Sede académica, México, 2011). Disponible en: https://www.researchgate.net/profile/Noe_Hernandez_Cortez/publication/317077357_El_discurso_ide ologico_de_la_politica_social_en_Venezuela_1989-2010_un_enfoque_postestructuralista/links/59245b96a6 fdcc444307600d/El-discurso-ideologico-de-la-politica-social-en-Venezuela-1989-2010-un-enfoque-postestruc turalista.pdf?origin=publication_detail

\section{Normatividad venezolana}

República Bolivariana de Venezuela, Constitución, 5.453 Gaceta Oficial, número extraordinario, 24 de marzo de 2000. Disponible en: http://www.cne.gov.ve/web/normativa_electoral/constitucion/indice.php

Venezuela, Decreto de la Presidencia de la República N³, 1999, mediante el cual se establece la realización de un referendo para que el pueblo se pronuncie sobre la convocatoria de una Asamblea Nacional Constituyente, 36.334 Gaceta Oficial, 2 de febrero de 1999. Disponible en: http://servicio.bc.uc.edu.ve/derecho/revista/idc22/22-27 .pdf

\section{Direcciones web}

Banco Mundial, Prevalencia de VIH, total (\% de la población entre 15 y 24 años de edad). Disponible en: https://dato s.bancomundial.org/indicador/SH.DYN.AIDS.ZS

Organización de Estados Americanos, OEA, Desigualdad e inclusión social en las Américas, 14 ensayos (OEA, Washington, 2014). Disponible en: https://www.oas.org/docs/desigualdad/libro-desigualdad.pdf

\section{Notas}

1 THOMAS HUMPHREY MARSHALL \& TOM BOTTOMORE, Ciudadania y clase social (Alianza Editorial, Madrid, 1998).

2 Varios autores discuten este tema, entre ellos: MARÍA LUCIANA AIN-BILBAO, Ser ciudadano en América Latina: un status conflictivo, 1 Iberoamérica Global, The Hebrew University of Jerusalem, 4, 3-13 (2008). GUILLERMO O'DONNELL, Hacia un Estado de y para la democracia, en Democracia/Estado/Ciudadania hacia un Estado de y para la democracia en América Latina, 
25-64 (RODOLFO MARIANI, coord., Programa de las Naciones Unidas para el Desarrollo, PNUD, Lima, 2008). LUCIANO NOSETTO, Variaciones latinoamericanas en torno al concepto de ciudadanía, 6 Revista de Filosofía Factótum, 77-97 (2009).

3 CARLOS BARBA-SOLANO, Reforma social y ciudadanía social en América Latina durante los años noventa: una perspectiva comparada, en Retos para la integración social de los pobres en América Latina, 51-84, 55 (CARLOS BARBA-SOLANO, comp., Colección Grupos de Trabajo, Consejo Latinoamericano de Ciencias Sociales, CLACSO, Buenos Aires, 2009).

4 Comisión Económica para América Latina y el Caribe, CEPAL, Panorama social de América Latina (CEPAL, Santiago de Chile, 2015). Banco Mundial, Informe anual 2015, 43 (Banco Mundial, Washington, D. C., 2015).

5 Banco Mundial, Informe anual 2015, 41-42 (Banco Mundial, Washington, D. C., 2015).

6 Para mayor precisión metodológica y claridad referencial, se tomarán 10 países referenciales: ocho suramericanos (Uruguay, Perú, Chile, Brasil, Venezuela, Colombia, Ecuador y Bolivia) y dos centroamericanos (Panamá y México, cabe destacar que este último está geográficamente ubicado entre Norte y Centroamérica).

7 Comisión Económica para América Latina y el Caribe, CEPAL, Panorama social de América Latina, 11 (CEPAL, Santiago de Chile, 2015). Banco Mundial, Informe anual 2015 (Banco Mundial, Washington, D. C., 2015).

8 ALICIA BÁRCENA, América Latina y el Caribe es la región más desigual del mundo ¿Cómo solucionarlo? Columna de opinión publicada en el marco de la reunión anual del Foro Económico Mundial (25 de enero de 2016).

9 Oxfam, Privilegios que niegan derechos. Desigualdad extrema y secuestro de la democracia en América Latina y el Caribe (2015).

10 GUILLERMO CRUCES, Protección social y sistemas de salud, en Los sistemas de saludy de protección social frente a los nuevos escenarios epidemiológicos y demográficos, 77-111 (Reunión de Expertos sobre Población y Pobreza en América Latina y el Caribe, 14 y 15 de noviembre 2006, Santiago, Chile, División de Población de la Comisión Económica para América Latina y el Caribe CELADE, con el auspicio del Fondo de Población de las Naciones Unidas, UNFPA, Santiago de Chile, 2006).

11 El primero de los patrones mencionados es característico de las sociedades desarrolladas (causas crónicas y degenerativas) y el segundo, se muestra asociado "con condiciones de vida socialmente deficientes (causas infecciosas y parasitarias), aunados a una mortalidad alta por accidentes y actos de violencia”. Organización Panamericana de la Salud, OPS-PAHO, Análisis de situación de salud en las Américas, 1999-2000, 21 Boletín Epidemiológico, 4, 2-3 (2000).

12 http://www.paho.org/hq/index Banco Mundial (http://www.bancomundial.org/indicador/)

13 ALEJANDRO AGUIRRE \& FORTINO VELA-PEÓN, Descenso y transición epidemiológica de la mortalidad infantil en América Latina y el Caribe, XLII Notas de Población, 110, 59-77, 61 (2015).

14 Oxfam, ibíd, 79. En este punto, solo se mencionan los países de los cuales se tienen datos.

15 Se considera gasto de bolsillo en salud el gasto privado en salud que hacen los hogares de manera directa.

16 De acuerdo a la misma fuente, la proporción de gasto de bolsillo erogado por los hogares como proporción de los gastos totales en salud en 2012 supera el 20\% del gasto total en salud (con excepción de Cuba y Uruguay); este porcentaje aumenta a niveles superiores al 50\% en países como Venezuela y Ecuador. MARINO J. GONZÁLEZ-R., Gasto de bolsillo en salud en América Latina (1995-2013): evolución e implicaciones de políticas (Trabajo de incorporación como miembro correspondiente nacional puesto 39 de la Academia Nacional de Medicina, Caracas, julio de 2016).

17 Los datos más elevados son los de Venezuela. En el caso brasileño no se reportan datos. Banco Mundial, Prevalencia de VIH, total (\% de la población entre 15 y 24 años de edad).

18 Organización de Estados Americanos, OEA, Desigualdad e inclusión social en las Américas, 14 ensayos (OEA, Washington, 2014).

19 AMARTYA SEN, ¿Por qué la equidad en salud?, 11 Revista Panamericana de Salud Pública, 5/6, 302-309 (2002).

20 LUIS GÓMEZ-CALCAÑO, Ciudadania, politica socialy sociedad civil en América Latina, 36 Cuadernos del Cendes, Centro de Estudios del Desarrollo, Universidad Central de Venezuela, 11-34, 20 (1997).

21 FRANCISCO ANTONIO ARIAS-MURILLO, Ciudadanía en el contexto democrático de América Latina, 3 Hallazgos, Revista de Investigaciones, 5, 151-165, 162 (2006). 
22 Si bien la Constitución de 1811 había elevado a rango constitucional el Decreto del 14 de agosto de 1810, de la Junta Suprema de Caracas que proscribía el comercio de esclavos en el país, la "institución de la esclavitud" como tal había mantenido vigencia.

23 Básicamente, hasta ese momento, el sufragio estaba restringido a los hombres venezolanos varones mayores de 21 años y alfabetos.

24 Cabe destacar que en marzo de 1946 la Junta Revolucionaria de Gobierno dictó el Estatuto Electoral para la Elección de Representantes a la Asamblea Nacional Constituyente, en el que estableció el sufragio universal, directo y secreto a todos los venezolanos mayores de 18 años, sin distinción de sexo, con la única excepción de los entredichos y los que cumpliesen condena penal, por sentencia firme que llevara consigo inhabilitación política.

25 Durante el siglo XX se dieron cuatro procesos constituyentes: el primero tuvo lugar en 1900, cuando se reunió la primera Asamblea Nacional Constituyente, que sancionó la Constitución el 23 de marzo de 1901, promulgada por el presidente Cipriano Castro el 29 de ese mismo mes y año; luego vino la Asamblea Nacional Constituyente de 1947, que sancionó la Constitución el 5 de julio de ese año. Pocos años después, en 1953, otra Asamblea Nacional Constituyente sancionó la llamada Constitución Nacional. Finalmente, en 1999, se reunió la última Asamblea Constituyente de ese siglo para promulgar la Constitución de 1999.

26 RICARDO COMBELLAS, Una Constitución para el futuro. El debate constitucional en Venezuela (Fundación Konrad Adenauer, Caracas, 1994).

27 En noviembre de 1948 se instauró una larga dictadura que convocó la Asamblea Nacional Constituyente de los Estados Unidos de Venezuela para sancionar una nueva Constitución que derogó expresamente la de 1947. Al hacerlo "cercenó teórica y prácticamente los derechos individuales y sociales" y no se dejó cabida a otra expresión de los derechos sociales. ABSALÓN MÉNDEZ-CEGARRA, Estado, politica social y trabajo social en la Venezuela actual, 246 (Universidad Central de Venezuela, Facultad de Ciencias Económicas y Sociales, Unidad de Publicaciones, Caracas, 1992).

28 LIGIA BOLÍVAR \& MAGALY PÉREZ-CAMPOS, El Sistema de Derechos Humanos en la Constitución de 1961 y Propuestas de Reforma, en El sistema politico venezolano: crisis y transformaciones, 33-129 (ÁNGEL EDUARDO ÁLVAREZ, coord., Universidad Central de Venezuela, Facultad de Ciencias Jurídicas y Políticas, Instituto de Estudios Políticos, Caracas, 1996).

29 MARIO DOS SANTOS, Estrategias de gobernabilidad en la crisis. Análisis comparado de las politicas de integración social en América Latina: fondos sociales de emergencia y tendencias a la focalización, en Desempleo estructural, pobreza y precariedad. Coordenadas y estrategias depolitica social en la Argentina y América Latina, 243-267, 245 (SUSANA PEÑALVA \& ALEJANDRO ROFMAN, comps., Centro de Estudios Urbanos y Regionales, CEUR, Ediciones Nueva Visión, Buenos Aires, 1996).

30 Para calmar esas tensiones se constituyó la Comisión para la Reforma del Estado (COPRE) que llevó a cabo reformas políticas significativas, dos de cuyos éxitos más resonantes fueron la descentralización y la elección directa, secreta y universal de las autoridades regionales y locales. Entre otros, EDITH MABEL CUNAARRO-CONDE, Modernización reflexiva y democratización en Venezuela, 20 Opción, Revista de Ciencias Humanas y Sociales, 43, 119-142 (2004). JORGE ERNESTO PÉREZ-LUGO, La necesaria reforma de los Sistemas de Salud en América Latina, 13 Gaceta Laboral, 1, 43-57 (2007). Comisión Presidencial para la Reforma del Estado, COPRE, Una politica social para la reafirmación de la democracia, volumen 8 (Editorial Arte, Caracas, 1989).

31 COPRE, ibíd.

32 LUIS GÓMEZ-CALCAÑO, Ciudadania, politica social ysociedad civil en América Latina, 36 Cuadernos del Cendes, Centro de Estudios del Desarrollo, Universidad Central de Venezuela, Cendes-UCV, 11-34, 20 (1997).

33 RAFAEL CALDERA, el candidato que a la postre ganó las elecciones, basó su campaña electoral en el documento Mi carta de intención con el pueblo de Venezuela, en el que denunciaba el deterioro de las condiciones de vida de la población y condenaba al gobierno de Carlos Andrés Pérez por la aplicación de las medidas económicas recomendadas por los organismos multilaterales. RAFAEL CALDERA, Mi carta de intención con el pueblo de Venezuela (Talleres Gráficos Joaquín Ibarra/Impresores, Caracas, 1993).

34 Entre esas políticas estuvieron el Plan de Solidaridad Social (PSS) y la Agenda Venezuela (1996-1998); esta última con un componente social de catorce programas destinados a los sectores más vulnerables.

35 JOSÉ E. MOLINA, Elproceso de consolidación de la hegemonia en Venezuela y sus consecuencias políticas, 9 Cuestiones Politicas, 73-81 (1992). 
36 TITO LACRUZ, Del antes al ahora: Balances de la política social en Venezuela, en Balance y perspectivas de la politica social en Venezuela, 111-184 (THAIS MAINGON, coord., Instituto Latinoamericano de Investigaciones Sociales, ILDIS, Centro de Estudios del Desarrollo, Universidad Central de Venezuela Cendes, Fondo de Población de las Naciones Unidas, UNFPA, Caracas, 2006).

37 LUIS GÓMEZ-CALCAÑO, La redefinición del Estado Social: el caso de Venezuela, en El cambio del papel del Estado en América Latina, 285-316 (MENNO VELLINGA, coord., Siglo XXI Editores, México, 1997).

38 Algunas de las razones esgrimidas fueron la crisis de representatividad por la que pasaba el sistema político venezolano y la pérdida de legitimidad de sus instituciones, frente a lo cual se proponía la consolidación de un Estado de Derecho cuya base jurídica permitiese la práctica de una democracia social y participativa. Venezuela, Decreto de la Presidencia de la República $N^{\circ} 3,1999$, mediante el cual se establece la realización de un referendo para que el pueblo se pronuncie sobre la convocatoria de una Asamblea Nacional Constituyente, 36.334 Gaceta Oficial, 2 de febrero de 1999.

39 República Bolivariana de Venezuela, Constitución, 5.453 Gaceta Oficial, número extraordinario, 24 de marzo de 2000.

40 NOÉ HERNÁNDEZ-CORTEZ, El discurso ideológico de la política social en Venezuela. 1989-2010: un enfoque postestructuralista, 196 (Tesis doctoral, Facultad Latinoamericana de Ciencias sociales, FLACSO, Sede académica, México, 2011).

41 AMARTYA SEN, Desarrollo y libertad (Editorial Planeta, Barcelona, 2000).

42 JO D'ELIA, Situación de la salud en Venezuela. La escasez se ha extendido a 1.200 medicamentos, 769 Revista SIC, 388-391 (2014). MARINO GONZÁLEZ, Las politicas de salud en Venezuela: alternativas para su mejoramiento (Instituto Latinoamericano de Investigaciones Sociales, ILDIS, Caracas, 2008). MARÍA NARANJO, La politica de salud en Venezuela durante el periodo 1999-2012. Aportes para su formulación e implementación, 12 Comunidad y Salud, 2, 80-86 (2014). Federación Médica de Venezuela, FMV, Diagnóstico del sector salud en Venezuela: estudios de las enfermedades emergentes y reemergentes (Ponencia central presentada en la LXIII Reunión Ordinaria de la Asamblea, realizada en Punto Fijo, estado Falcón, 27 al 31 de octubre de 2008). CARLOS WALTER, A Strange Form of Declaring a Health Emergency: The Case of Venezuela, 55 World Medical Journal, 4, 157-159 (2009).

43 En 2009, el entonces presidente de la República Hugo Chávez, durante un Consejo de Ministros ampliado realizado en el estado Guárico alertó sobre la grave situación de la salud en Venezuela y declaró la emergencia en el área: "En lo social tenemos una emergencia: la salud. Declarémonos en emergencia todos". http://www.eluniversal.com/2009/09/24/pol

44 Una noticia de prensa de la fecha señalaba: "informó este sábado [el vicepresidente Maduro] que el Gobierno Bolivariano decidió intervenir el Hospital Periférico de Coche, en Caracas, por irregularidades constatadas en este centro asistencial: \#Hemos decidido intervenir el hospital de Coche para ir a un proceso profundo de reestructuración [...] será revisado lo relativo a la infraestructura, dotación de equipos y de personal, ya que se requiere médicos de diversas especialidades, sobre todo pediatras... \#” (http://www.correodelorinoco.gob.ve/caracas/gobierno-nacional-intervino-hospital-periferico-coche).

45 Programa Venezolano de Educación-Acción en Derechos Humanos, PROVEA, Informe Anual 2013: Situación de los derechos bumanos en Venezuela, 197 (2013).

46 Sobre este asunto es pertinente señalar que el Área de Desarrollo y Salud del Cendes-UCV, del que forma parte la autora de este trabajo, sostiene que en Venezuela no se puede hablar de un sistema nacional de salud, ya que en realidad lo que existe es una multiplicidad de órganos y prestadores de servicios, públicos y privados, que se manejan de manera inconexa y fragmentada, sin la unicidad de criterios que debe haber para hablar de un sistema, como cuerpo orgánico y estructurado.

47 Alrededor de 15.000 profesionales de la medicina han renunciado a sus puestos de trabajo en el sector público; de los cuales el $46 \%$ trabaja en organismos privados, mientras que el déficit del personal de enfermería podría estar alcanzando el 60\%. JO D'ELIA, Situación de la salud en Venezuela. La escasez se ha extendido a 1.200 medicamentos, 769 Revista SIC, 388-391 (2014).

48 De acuerdo con un estudio realizado por un equipo interdisciplinario, bajo los auspicios de la Cámara de Comercio de Maracaibo (2011), para 2010, en el país hubo un brote de mal de Chagas, producto de lo cual falleció una persona; por otra parte, indica esa misma fuente, Venezuela llegó a ocupar el primer lugar en América en casos de dengue, que aumentaron en un 85\%, mientras que los de malaria en un $138 \%$, en comparación con el mismo período del año anterior. Cámara de Comercio de Maracaibo, Reflexiones sobre el Sistema de Salud Venezolano, Trabajo elaborado por un equipo interdisciplinario bajo el auspicio de la Cámara de Comercio de Maracaibo, 8 (2011). 
49 Sociedad Venezolana de Salud Pública, SVSP \& Red Defendamos la Epidemiología Nacional, Pequeñas picaduras, grandes amenazas. Breve relato de éxitos, fracasos y amenazas de la luch a contra las enfermedades vectoriales en Venezuela, 3, Día Mundial de la Salud de 2014, 7 de abril de 2014, dedicado al control de las enfermedades transmitidas por vectores.

50 Ministerio del Poder Popular para la Salud, MPPS, Boletin epidemiológico (2010). Ministerio del Poder Popular para la Salud, MPPS, Boletín epidemiológico (2014).

51 Federación Médica de Venezuela, FMV, Diagnóstico del sector salud en Venezuela: estudios de las enfermedades emergentes y reemergentes, 144 (Ponencia central presentada en la LXIII Reunión Ordinaria de la Asamblea, realizada en Punto Fijo, estado Falcón, 27 al 31 de octubre de 2008).

52 Banco Mundial, Informe anual (2015).

53 Programa Venezolano de Educación-Acción en Derechos Humanos, PROVEA, Informe Anual 2013: Situación de los derechos humanos en Venezuela, 197 (2013).

\section{Licencia Creative Commons CC BY 4.0}

Para citar este artículo/To cite this article: Delgado Blanco, Andy del Valle, El derecho a la salud como dimensión de la ciudadanía social en América Latina y Venezuela, 137 Vniversitas, xxx-xxx (2018). https:// doi.org/10.11144/Javeriana.vj137.dsdc 